\title{
RESOLUTIVIDADE DA FISIOTERAPIA NA ATENÇÃO BÁSICA À SAÚdE (AB): A PERCEPÇÃO DE FISIOTERAPEUTAS
}

\author{
RESOLUTENESS OF THE PHYSIOTHERAPY IN PRIMARY HEALTH CARE (PHC): \\ A PHYSIOTHERAPIST PERCEPTION
}

\author{
Ana Jéssica de Lima \\ Natália Rennó Lemes Aley \\ Curso de Fisioterapia \\ Universidade Federal de Alfenas \\ Geraldo Eduardo Guedes de Britto \\ Departamento de Fisioterapia \\ Universidade Federal da Paraíba

\section{Sueli Leiko Takamatsu Goyatá \\ Curso de Enfermagem \\ Universidade Federal de Alfenas} \\ Silvia Lanziotti Azevedo da Silva \\ Curso de Fisioterapia \\ Universidade Federal de Alfenas
}

\author{
Contato \\ Silvia Lanziotti Azevedo da Silva \\ Avenida Jovino Fernandes Salles, 2600 \\ Bairro Santa Clara \\ Alfenas-MG \\ CEP: $37130-000$ \\ E-mail: silviafisiojf@yahoo.com.br
}

\section{RESUMO}

A atuação da fisioterapia na Atenção Básica à Saúde $(A B)$ é um processo em construção que enfrenta desafios, visto que o fisioterapeuta é formado para a reabilitação. Este estudo teve como objetivo compreender a percepção dos fisioterapeutas atuantes na $A B$ sobre resolutividade de suas ações e possíveis mudanças com a utilização de protocolo de encaminhamentos na organização do serviço. Utilizou-se metodologia qualitativa e descritiva, com análise de conteúdo. Os profissionais entrevistados apontaram a resolutividade das ações a partir de duas perspectivas antagônicas: os fisioterapeutas que consideram a prevenção, a promoção e a educação em saúde como

práticas inerentes à Fisioterapia avaliaram a resolutividade das ações como boa, e aqueles que consideram apenas o tratamento de doenças apontaram-na como baixa. Por fim, é importante conscientizar os fisioterapeutas da importância da funcionalidade na definição do acesso a serviços mais complexos, desde a sua formação, buscando maior resolutividade na $A B$. $O$ investimento na formação desse profissional é fundamental para que, dentro de sua atuação, ele valorize menos a técnica e mais as posturas humanizadas, o diálogo e a funcionalidade de seus pacientes, recursos de grande importância para a atuação dentro do primeiro nível da rede de atenção.

Palavras-chave: Fisioterapia. Atenção Primária à Saúde. Entrevista. Análise Qualitativa. 


\section{ABSTRACT}

Physical therapy action in Primary Heath Care (PHC) is a process under construction and faces many challenges, since the physiotherapist is regarded as a rehabilitation professional. This study aimed to understand the perceptions of physiotherapists acting in PHC on the resoluteness of their actions and possible changes in case of using a referral protocol in the service organization. Qualitative and descriptive methodology were used, with content analysis. The professionals interviewed noted the resoluteness of the actions from two opposing perspectives: professionals who consider prevention, promotion and health education as practices inherent to Physical the- rapy reviewed the resoluteness of actions as good. However, those who consider only the treatment of diseases reviewed the resoluteness of actions as bad. Finally, it is important to make physiotherapists aware of the importance of functionality in defining access to more complex services, since their training, seeking a higher resolution in PHC. Investment in training such professionals is essential so that, within their practice, they value less the technic and more humane attitudes, the dialogue and the functionality of their patients, very important resources in the action within the first level of care network.

Keywords: Physical Therapy Specialty. Primary Health Care. Interview. Qualitative Analysis.

\section{INTRODUÇÃO}

A inserção da fisioterapia na Atenção Básica à Saúde $(A B)$ é um processo em construção. Assim, enfrenta alguns desafios, consequentes do caráter exclusivamente reabilitador da profissão, que consolidou suas práticas assistenciais no tratamento de doenças e suas sequelas no âmbito ambulatorial e hospitalar, o que era fortemente refletido nos currículos de formação de trabalhadores fisioterapeutas ${ }^{1}$. Com base nesse contexto, historicamente, a fisioterapia não se inseriu na $A B$, o que pode, inclusive, ter ocasionado a restrição do acesso da população a esse serviço².

Em especial em 2002, com as novas Diretrizes Curriculares Nacionais (DCN) do Curso de Fisioterapia, o objeto de trabalho da fisioterapia passou por redefinições e, mesmo que de maneira incipiente, introduziu a promoção de saúde e a prevenção de agravos como área de atuação, passando, assim, a compor grades curriculares nos cursos de fisiotera$\mathrm{pia}^{3}$. No que se refere à inserção na $A B$, a presença do fisioterapeuta nesse nível de atenção à saúde foi garantida por meio da Portaria GM 154/2008, que criou o Núcleo de Apoio à Saúde da Família (NASF), equipe multiprofissional que dá apoio as ações da Equipe de Saúde da Família (ESF)4.

Atualmente, de acordo com a Organização Mundial de Saúde (OMS), o declínio dos problemas agudos e ascensão das condições crônicas estão causando falhas nos sistemas de saúde em todo o mundo5. Devido à fragmentação da organização destes, o conjunto de pontos de atenção são isolados e não se comunicam, sendo, assim, incapazes de fornecer uma atenção contínua à população. Com sistema fragmentado, não há comunicação entre $A B$ e a atenção especializada à saúde, e esses dois também não se comunicam com a atenção hospitalar, tampouco com os sistemas de apoio. Dessa forma, a $A B$ não exerce sua função de comunicação, coordenando o cuidado, inclusive na Fisioterapia ${ }^{5}$.

As Redes de Atenção à Saúde surgem para enfrentar essas condições impostas pelos sistemas fragmentados. Elas são organizações poliárquicas pelos conjuntos de serviços de saúde, que estabelecem ações cooperativas e interdependentes, além de oferecer uma atenção contínua e integral à população. Além disso, oferecem continuidade aos níveis de atenção básica, especializada e hospitalar, realizando atenção integral com intervenções promocionais, preventivas, curativas, cuidadoras, reabilitadoras e paliativas, funcionando sob gerenciamento da $\mathrm{AB}^{6}$.

É essencial que a $A B$ apresente alta resolutividade, que por sua vez depende da capacidade clínica, dos cuidados de suas equipes e da articulação dela com outros pontos da rede de saúde ${ }^{8}$. Dessa forma, a regulação da assistência à saúde é fundamental para ordenar o acesso às ações e serviços, selecionando as consultas e procedimentos apenas quando apresentarem indicações clínicas para realizá-los, e como consequência, reduzir os encaminhamentos desnecessários ${ }^{8}$.

Uma alternativa para organizar o processo de referenciamento de usuários da $A B$ para serviços especializados é a adoção de protocolos de encaminhamentos. Estes precisam ser articulados a processos que aumentem a capacidade clínica das equipes e propiciem comunicação entre Unidades Básicas de Saúde (UBS) e serviços especializados. As informações clínicas exigidas nesses protocolos devem ser sucintas, objetivas, necessárias e suficientes para a prática da regulação ${ }^{8}$. 
Dentro do serviço de fisioterapia, os próprios profissionais devem aplicar os protocolos, seja em centrais de regulação ou na própria UBS onde atuam. Entretanto, isto não é comum; e os fisioterapeutas desconhecem a inserção dessa proposta em sua rotina de trabalho.

Destarte, o objetivo deste estudo foi compreender a percepção dos fisioterapeutas que atuam na $A B$ em um município do sul de Minas Gerais sobre a resolutividade de suas ações, ante a sua formação, e a aceitação de alguma possibilidade de mudanças no processo de trabalho com o uso de protocolos de encaminhamento baseados na valorização da funcionalidade na rotina de atuação.

\section{MÉTODO}

T rata-se de um estudo qualitativo e descritivo, desenvolvido em uma cidade do sul de Minas Gerais. Na época do estudo, o município possuía 15 ESF, das quais 14 localizadas na zona urbana e 1 na zona rural, e ainda 2 UBS Tradicionais. De acordo com estimativa populacional do Instituto Brasileiro de Geografia e Estatística (IBGE), o município possui 79.222 habitantes no ano de 2016, quando foi realizado o estudo. No que se refere à organização da $A B$, em torno de $70 \%$ da população recebe cobertura da ESF, e o restante tem como referência as UBS. Cinco fisioterapeutas prestam suporte às $14 \mathrm{ESF} e$ às 2 UBS, revezando sua atuação entre elas ao longo de jornada de trabalho semanal de 30 horas. A zona rural não possui cobertura fixa dos fisioterapeutas, havendo atendimento somente em caso de procura pelo serviço para o usuário.

Optou-se por entrevistar os cinco fisioterapeutas que atuam na $A B$ por meio de uma entrevista semiestruturada autoaplicada, respondida por escrito, em dois momentos, antes e após a implementação de um protocolo (Anexo 1), para ordenação dos encaminhamentos da $A B$ para a atenção especializada na rede municipal. A elaboração do questionário respeitou as recomendações da literatura especializada, e a autoaplicação possibilitou aos fisioterapeutas mais privacidade ao responder à entrevista. As perguntas procuraram não direcionar as respostas dos entrevistados, eram de fácil interpretação e claras. Em caso de dúvida, os fisioterapeutas podiam solicitar esclarecimentos do pesquisador, que aguardava no local da avaliação o preenchimento do questionário. Antes de responder às perguntas, os fisioterapeutas assinaram o Termo de Consentimento Livre e Esclarecido (TCLE), e todos os profissionais que compunham o universo no estudo concordaram em participar, não havendo recusas ou perdas.
O primeiro momento do estudo aconteceu no mês de fevereiro de 2016. O instrumento de coleta de dados apresentou cinco questões estruturadas que visaram caracterizar os fisioterapeutas das ESF quanto ao gênero, idade, tempo de formação e de atuação na $A B$, se possuíam especialização, incluindo Saúde da Família, e se já atuaram em outras áreas. As questões abertas da primeira rodada de entrevistas foram: "Como você define a resolutividade da Atenção Básica para a fisioterapia?"; "O que você considera como prioridade para encaminhamento para o serviço especializado de fisioterapia?"; "O que você considera capaz de resolver na Atenção Básica?"; "Quais as principais patologias de base apresentadas pelos pacientes que buscam a Atenção Básica?"; "O que você considera importante conter em um protocolo aplicado na Atenção Básica para encaminhamento para a Atenção Especializada?".

A segunda fase do estudo ocorreu em um período de três meses após a implementação de um protocolo piloto de encaminhamento (julho de 2016). Essa segunda rodada de entrevista foi composta pelas seguintes perguntas: "O que você considera como prioridade de encaminhamento para o serviço especializado de Fisioterapia?"; "A aplicação do protocolo mudou a sua percepção dos itens que devem ser considerados no momento de encaminhar o paciente para a atenção especializada?"; "Você acha que o protocolo ajudou a tornar a atenção básica mais resolutiva para a Fisioterapia?"; "Você considera que o protocolo foi capaz de discriminar corretamente quem deveria ser encaminhado para a Atenção Especializada?"; "Você tem alguma sugestão de mudança para o protocolo?". A pergunta "O que você considera como prioridade para encaminhamento para o serviço especializado de fisioterapia?" foi mantida nos dois momentos para verificação se haveria mudança na perspectiva dos profissionais acerca da resolutividade da $A B$ e das reais necessidades de encaminhamento. Foram selecionadas para a análise e interpretação as perguntas cujas respostas apresentaram variação entre os entrevistados e contribuíram para percepção da resolutividade pelos fisioterapeutas. Aquelas que as respostas foram iguais ou muito sucintas foram excluídas da análise final.

O protocolo de elegibilidade e encaminhamento (Anexo 1) para a fisioterapia foi elaborado com embasamento teórico na Classificação Internacional de Funcionalidade, Incapacidade e Saúde $(\mathrm{CIF})^{8}$, com o objetivo de alterar o foco da doença no acesso ao serviço especializado, valorizar a funcionalidade e o impacto da condição de saúde no cotidiano do usuário. O protocolo é divido em cinco sessões: dados sobre o diagnóstico, dor, incapacidade, cognição e necessi- 
dade ou presença de cuidador. Essas sessões foram baseadas em domínios da CIF: condição de saúde, estrutura e função do corpo, atividades, participação, fatores pessoais e fatores ambientais. Os dados sobre o diagnóstico e a dor referem-se à condição de saúde e estrutura e função do corpo, a incapacidade relaciona-se com as atividades e participação, com a cognição com os fatores pessoais e com o item cuidador com os fatores ambientais. Cada item da proposta do protocolo era pontuado, e, ao final, poderia ser alcançada uma pontuação máxima de 26 pontos, sendo que quanto maior a pontuação, maior a necessidade de acesso ao serviço especializado de fisioterapia. Uma estimativa inicial de ponto de corte foi feita, em que usuários que pontuassem mais de nove pontos eram encaminhados. O protocolo deveria ser aplicado pelos fisioterapeutas da $A B$, que foram entrevistados nesta pesquisa.

Para a análise do conteúdo das entrevistas, foi realizada uma leitura precisa, crítica e exaustiva do material produzido pelas perguntas norteadoras9. Em relação à garantia de anonimato dos profissionais envolvidos na pesquisa, foi utilizado um código, no qual cada fisioterapeuta foi denominado por um número, sendo os números escolhidos 1, 2, 3, 4 e 5. Cada trecho destacado nas falas foi identificado pela letra maiúscula "T", seguida pelo número do fisioterapeuta ao qual ele se refere. A metodologia deste estudo visou evidenciar o sentido das informações colhidas, utilizando técnicas de pesquisa que favorecem a comparação e interpretação dos resultados ${ }^{9,10}$. O tratamento dos dados foi realizado por meio da análise do conteúdo, conduzida por um olhar interpretativo do material produzido. Para isso, as entrevistas passaram pelos processos de pré-análise (leitura flutuante do material final e elaboração de indicadores iniciais), exploração do material (identificação das unidades de registro), tratamento dos resultados, inferência e interpretação (condensação e definição das informações para análise reflexiva e crítica dos resultados). Além disso, foram feitas análises de frequência em que as informações mais objetivas foram repetidas pelos fisioterapeutas em cada uma das perguntas.

O estudo foi aprovado pelo Comitê de Ética e Pesquisa, parecer $N^{\circ} 1.125 .782$. O TCLE foi entregue aos participantes da pesquisa, em duas vias de igual conteúdo, para assinatura. Esse termo continha todas as explicações detalhadas sobre a pesquisa.

\section{RESULTADOS E DISCUSSÃO}

amostra foi composta por cinco fisioterapeutas,
cujas características estão descritas na Tabela 1:

Tabela 1. Caracterização dos Fisioterapeutas participantes da pesquisa

\begin{tabular}{|c|c|c|c|c|c|c|}
\hline Fisioterapeuta & Idade ${ }^{*}$ & Sexo $^{*}$ & $\begin{array}{l}\text { Tempo } \\
\text { de graduação* }\end{array}$ & $\begin{array}{l}\text { Tempo que } \\
\text { atua na } \mathrm{AB}^{\star}\end{array}$ & Especialização $^{*}$ & $\begin{array}{c}\text { Já atuou em outra } \\
\text { área? Qual?* }\end{array}$ \\
\hline 1 & 40 & Feminino & 16 anos & 12 anos & $\begin{array}{l}\text { Gestão em Saúde } \\
\text { Pública, Acupuntura, } \\
\text { Pediatria }\end{array}$ & $\begin{array}{l}\text { Sim, Apae e clínica } \\
\text { particular }\end{array}$ \\
\hline 2 & 38 & Feminino & 15 anos & 3 anos & $\begin{array}{l}\text { Geriatria, Acupuntura, } \\
\text { Neurologia }\end{array}$ & $\begin{array}{l}\text { Sim, ortopedia, } \\
\text { cardiorrespiratória e } \\
\text { neurologia }\end{array}$ \\
\hline 3 & 32 & Feminino & 10 anos & 8 anos & Acupuntura & $\begin{array}{l}\text { Sim, hospital e } \\
\text { estética }\end{array}$ \\
\hline 4 & 30 & Masculino & 7 anos & 6 anos & $\begin{array}{l}\text { Acupuntura, Saúde da } \\
\text { Família, Atividades } \\
\text { Físicas e Esportes para } \\
\text { Pessoas com Deficiência }\end{array}$ & $\begin{array}{l}\text { Sim, acupuntura e } \\
\text { atendimento } \\
\text { particular }\end{array}$ \\
\hline 5 & 25 & Feminino & 2 anos e meio & 5 meses & $\begin{array}{l}\text { Residência em Saúde } \\
\text { da Família, UTI adulto }\end{array}$ & Não \\
\hline
\end{tabular}

Informações obtidas por questionário estruturado aplicado com os participantes antes das perguntas norteadoras. 
Os resultados da análise de conteúdo das entrevistas apontaram a temática principal, resolutividade da Fisioterapia na $A B$, a partir de duas perspectivas antagônicas. Esse resultado heterogêneo é sugestivo de uma confusão conceitual e prática acerca da finalidade do trabalho do Fisioterapeuta nesse nível de atenção em saúde.

Apenas um profissional considerou como práticas inerentes à Fisioterapia no âmbito da $A B$ aquelas associadas à prevenção, promoção e educação em saúde, avaliando assim a resolutividade das ações como boa. Sinalizou também a materialização de sua prática a partir de intervenções em grupos operativos e da avaliação de casos seguidas de orientações ou encaminhamentos para nível secundário. A fala a seguir ilustra essa avaliação.

\section{A Fisioterapia na Atenção Básica torna-se resolutiva trabalhando na prevenção, pro- moção e educação em saúde através da formação de grupos operativos e durante a consulta para avaliação e triagem dos pa- cientes para resolver seu caso. Estes casos podem ser resolvidos na Unidade ou serem encaminhados para outros níveis de aten- ção quando necessário. (T5)}

O fisioterapeuta identificado como T5 se destaca da amostra por ser mais jovem e ser egresso de um programa de Residência em Saúde da Família, já dentro de um modelo de ensino voltado para práticas em $A B$. Esse fato ressalta a importância da formação desse profissional dentro de uma perspectiva de atuação que representa um importante avanço na concepção das práticas da Fisioterapia, ao incorporar as ações coletivas, de educação em saúde, na compreensão das redes do Sistema Único de Saúde (SUS), com a $A B$ ordenando e coordenando o fluxo de usuários nos serviços de atenção fisioterapêutica. Essa formação multiprofissional propicia maior conscientização e preparo para a prática, contribui para modificações no processo de trabalho e para a educação permanente dos profissionais. Dessa forma, este tipo de capacitação é de extrema relevância para a formação do fisioterapeuta que deseja atuar em $A B$, proporcionando uma visão ampla e experiência diversificada11. Cabe destacar que esse "novo" olhar da Fisioterapia não exclui as práticas curativistas, porém, elas são mais adequadas à realidade das Unidades de Saúde da Família (USF) e trazem novas ferramentas de trabalho. A inserção do fisioterapeuta em programas de residência multipro- fissional em saúde da família mostrou-se de grande valor no desenvolvimento de uma atuação mais humanizada e inserida nos princípios da $A B$, dentro de um trabalho em equipe e com maior resolutividade ${ }^{12}$.

Os outros quatro profissionais que consideram apenas o tratamento de doenças apontaram como baixa a resolutividade da fisioterapia na AB. Atribuíram isso às dificuldades dos usuários em seguir orientações prestadas, número insuficiente de profissionais fisioterapeutas e falta de espaço físico e equipamentos apropriados. Além disso, apontaram ainda a insuficiência de vagas no setor secundário, o que fragiliza o itinerário terapêutico dos usuários dos serviços de Fisioterapia.

Sem resolutividade, por falta de profissionais e espaço físico adequado. (T2)

Resolutividade baixa, pois orientam os usuários e eles não seguem as orientações. Poucas vagas nas clínicas para os encaminhamentos. (T3)

Poderíamos ter equipamentos básicos da Fisioterapia para atuarmos com melhor resolutividade [...], ou seja, maior investimento em equipamentos e aparelhos [...]. (T4)

Essa perspectiva de prática fisioterapêutica se encontra baseada no modelo assistencial historicamente construído por essa profissão, de uma abordagem individual, fragmentada, tecnicista, concentrada principalmente no nível secundário e dependente de recurso tecnológico13. Os fisioterapeutas aos quais pertencem esses últimos relatos estão inseridos há mais tempo no mercado de trabalho, estando mais suscetíveis à influência do modelo de atenção vigente na maioria dos serviços de fisioterapia. Assim, a atuação desses profissionais vem sendo contemplada na prática do modelo biomédico, com atividades predominantemente reabilitadoras. Suas ações, porém, deveriam estar voltadas ao modelo de atenção integral à saúde ${ }^{13}$.

Os fisioterapeutas que não consideraram a fisioterapia na $A B$ resolutiva têm maior tempo de formação e atuação, sendo tal formação anterior a 2002, quando entraram em vigor as novas DCN do Curso de Fisioterapia. Tais Diretrizes modificaram a antiga proposta do curso de Fisioterapia, que valorizava a técnica e a especialização do profissional. Elas estabelecem uma formação generalista, humanista, com capacidade crítica e reflexiva, e ainda de- 
terminam que os profissionais devem realizar seus serviços com alta qualidade e dentro dos princípios da ética, com ações de prevenção, promoção, proteção e reabilitação da saúde, tanto em nível individual quanto coletivo ${ }^{3,14}$. Essa nova proposta prepara melhor o fisioterapeuta para atuar na $A B$; e a maioria dos que responderam a esta entrevista não apresentaram tal formação, o que pode explicar suas opiniões sobre a resolutividade de suas ações.

A AB presume a resolutividade das necessidades de saúde como uma esfera que extrapola a intervenção curativa e reabilitadora, por meio da promoção da saúde, prevenção de doenças e educação em saúde ${ }^{15}$. A inserção da fisioterapia nesse nível de atenção é recente. Uma das principais dificuldades encontradas na inserção do fisioterapeuta diz respeito à formação inicial e à criação da profissão, que priorizam ação curativa, caráter reabilitador e práticas concentradas nos níveis secundário e terciário de atenção. A partir disso, durante muito tempo, os serviços de fisioterapia apresentaram pouca inserção na $A B$, determinando uma grande dificuldade de acesso da população a esse serviço².

A tradição do uso de equipamentos como uma identidade profissional do fisioterapeuta é outro aspecto que dialoga com a percepção de baixa resolutividade. Essa utilização de tecnologias em relação ao seu trabalho remonta ao modelo assistencial, curativista e tecnicista ${ }^{13}$. Além disso, existe a valorização da sociedade atual acerca da tecnologia, das especialidades e das práticas direcionadas à assistência curativa. Essas características dificultam o desenvolvimento de ações de prevenção e promoção de saúde, sendo estas comuns na $A B$ e presentes em um novo modelo de atenção à saúde. Para desenvolver atividades desse nível, os profissionais devem estar bem preparados e informados sobre as políticas de saúde e particularmente sobre o SUS ${ }^{16}$. Por essa razão, a capacitação do profissional para a ação preventiva e educativa é de extrema importância para a comunidade em que atua, contribuindo para a melhora dos serviços prestados ${ }^{16}$. Um estudo qualitativo evidenciou que, na percepção de usuários de USF, o fisioterapeuta é ainda percebido como profissional de reabilitação, mas, em algumas falas, sua atuação na promoção de saúde foi identificada ${ }^{17}$. Essa perspectiva mostra que é possível o crescimento da atuação desse profissional nesse novo campo.

A análise da frequência das respostas dos entrevistados apontou informações sobre casos mais atendidos e quais, segundo eles, demandam encaminhamento para o setor secundário. A maioria dos entrevistados considerou que alterações osteomusculares, tais como dores na coluna e alterações posturais, são as principais demandas para eles em sua atuação na $A B$, sendo aquelas que podem ser resolvidas nesse nível de atenção.

Os fisioterapeutas entrevistados consideraram pós-operatórios e casos cirúrgicos mais complexos os que demandavam atenção no nível secundário, necessitando de encaminhamento. Essa pergunta foi novamente realizada na segunda rodada de entrevistas, sendo reafirmada a necessidade de encaminhamento para o serviço especializado casos de pós-operatório e casos agudos. Na segunda entrevista, foram mencionados casos menos comuns, como Acidente Vascular Cerebral recente (60\%), crianças $(40 \%)$ e problemas uroginecológicos $(20 \%)$.

A doença como determinação dos encaminhamentos demonstra uma abordagem curativa, em que os problemas de saúde são restritos aos aspectos fisiopatológicos, distanciando os determinantes socioeconômicos preponderantes no processo saúde e doença ${ }^{18}$. A atuação profissional deve ser separada dessa dicotomia pelo fato desse conceito não ser apropriado para entender o objetivo de trabalho no campo de Saúde Coletiva, que carrega o paradigma da determinação social da saúde e da doença como um processo relacional ${ }^{18}$.

Após a segunda rodada de entrevistas, não foi evidenciada mudança na percepção dos fisioterapeutas, o que reflete o desconhecimento da atuação em Saúde Pública, evidenciado, talvez, uma falta de capacitação, qualificação, monitorização dos serviços e valorização dos profissionais desde sua entrada no sistema ${ }^{19}$. Dentro da Lei 8.080 , estão previstos programas de educação permanente para o aperfeiçoamento dos profissionais do SUS ${ }^{20}$. Os processos de educação dos trabalhadores da saúde devem considerar que as necessidades de formação e desenvolvimento dos trabalhadores sejam pautadas pelas necessidades de saúde das pessoas e populações. Os processos de educação permanente em saúde têm como objetivos a transformação das práticas profissionais e da própria organização do traba$\mathrm{Iho}^{21}$. A educação permanente em saúde, entretanto, é um desafio para os profissionais, uma vez que não é ainda rotina na maioria dos serviços, sua discussão é mais forte na Enfermagem e, muitas vezes, suas iniciativas pouco ajudam no desenvolvimento do processo de trabalho. É preciso desenvolver as propostas para obter melhores resultados ${ }^{22,23}$.

Sobre protocolo de encaminhamento elaborado para nortear esse processo, antes do início de 
sua aplicação, os fisioterapeutas consideraram importante que o instrumento fosse capaz de discriminar entre casos agudos e crônicos e outros dados referentes ao diagnóstico, sendo que somente um fisioterapeuta destacou a capacidade funcional como ponto a ser inserido. Após terem contato com a proposta, os fisioterapeutas, de forma geral, não consideram que o protocolo foi capaz de auxiliar na determinação dos casos que deveriam ser encaminhados. Todavia, observa-se uma contradição, já que $80 \%$ dos profissionais relataram que o protocolo foi capaz de aumentar a resolutividade da $A B$ por determinar quem deveria ser mantido neste nível. Na verdade, após a inserção da proposta do protocolo, houve um esforço da gestão e mudança no processo de trabalho do fisioterapeuta, que começou a acolher todos os casos encaminhados, e estes não foram mais encaminhados diretamente para o serviço especializado. Acredita-se então que os fisioterapeutas consideraram o aumento da resolutividade baseado no contato com o usuário que passou a ocorrer mais frequentemente, e não à aplicação da ferramenta em si.

Alguns fisioterapeutas (60\%) julgaram que o protocolo está voltado para a ortopedia, o que não faz sentido, visto que ele foi criado com embasamento na ideia central da CIF, e não em especialidades. Tal visão tradicional não foi modificada pela nova proposta, como relatado em $80 \%$ das entrevistas. Essa percepção dos fisioterapeutas pode estar relacionada com a criação recente da CIF, que foi aprovada em 2001 e traduzida para a língua portuguesa em $2003^{24}$. Nessa época, a maioria dos profissionais entrevistados já havia concluído a formação. Além disso, nem sempre a CIF é um conteúdo abordado de forma aprofundada durante a graduação. Geralmente, o assunto é exposto aos alunos de forma superficial. Em consequência disso, a maioria dos profissionais que começam a utilizá-la apresentam limitações em relação a sua aplicabilidade. A ausência de capacitações específicas sobre a CIF e sua restrita aplicação nas práticas clínicas resultam em limitado conhecimento e compreensão dessa ferramenta ${ }^{25}$.

A concepção da importância da capacidade funcional foi citada por apenas um profissional. O que deveria ser diferente, já que, na atualidade, os modelos de reabilitação refletem uma mudança de paradigma e definem saúde em termos mais amplos, indicando que fatores sociais, psicológicos e ambientais contribuem para a saúde e a qualidade de vida ${ }^{26}$.
A adesão de um modelo de funcionalidade e incapacidade humana possibilitaria ao fisioterapeuta, em seus procedimentos de avaliação e de intervenção, considerar um perfil funcional individualizado para cada paciente ${ }^{27}$, buscando condutas específicas ${ }^{28}$. O conceito ampliado de saúde na avaliação fisioterapêutica é capaz de demonstrar que uma mesma patologia, diagnosticada em diferentes sujeitos, não causará o mesmo impacto, e este não se restringe apenas ao nível de estrutura e função do corpo. Sendo assim, a avaliação deve ser voltada para o paciente, considerando o mesmo grau de importância todas as dimensões da saúde, incluindo as atividades e a participação social ${ }^{29}$. Essa percepção equivocada pode ser novamente relacionada com uma formação especialista e fragmentada do fisioterapeuta. Este problema se reflete em seu perfil acadêmico, voltado apenas para o processo de reabilitação. Ademais, como consequências disso, os profissionais estão sendo formados para o uso intensivo de tecnologia e para especialização, o que, como já foi dito, não é um perfil adequado para a atuação na $A B^{30}$.

O propósito do protocolo de encaminhamento é orientar o fluxo dos usuários, reduzindo os encaminhamentos desnecessários, selecionando o acesso de pacientes que realmente apresentem indicação clínica. Esse mecanismo de filtro auxilia na resolutividade da $A B$, além de ampliar o acesso da população aos serviços de saúde e melhorar a qualidade desses serviços8. Contudo, a aplicação de protocolos de triagem ainda não é algo disseminado na prática clínica dos fisioterapeutas. Isso revela a necessidade de capacitação por meio de programas de educação permanente, bem como a importância do envolvimento desses profissionais na adaptação do protocolo.

O tempo de três meses do início da aplicação do protocolo para a avaliação de seu impacto na resolutividade do serviço de fisioterapia na percepção dos fisioterapeutas pode ser considerado pequeno. Entretanto, o objetivo central do estudo não foi avaliar o protocolo, mas sim a percepção da resolutividade pelos profissionais fisioterapeutas atuantes na $A B$ de forma geral e o impacto que ela poderia sofrer diante da aplicação de uma nova proposta, ante sua formação já discutida. Esta questão revelou a resistência dos profissionais a propostas e ferramentas diferentes de trabalho, levando à dificuldade na incorporação de práticas pioneiras. 


\section{CONCLUSÃO}

$\mathrm{O}$ resultados da pesquisa chamam a atenção para a importância de conscientizar os fisioterapeutas da relevância da consideração da funcionalidade na definição do acesso a serviços com maior nível de complexidade, para que se alcance resolutividade ideal para as condições de saúde dos usuários. Outro aspecto que se tornou evidente neste estudo foi a necessidade de uma formação profissional direcionada para a atuação na $A B$ e a indispensabilidade da inserção de atividades voltadas para esse nível de atenção, visto que ficou salientada uma prática tecnicista. Uma alternativa para aqueles profissionais já inseridos no mercado, em busca de uma reformulação de sua atuação, é a Educação Permanente em Saúde, para qualificação voltada para a prática profissional e redução da visão tecnicista e especializada. A prática fisioterapêutica é muito relevante na $A B$, já que o profissional fisioterapeuta possui potencialidades para trabalhar com a criatividade e desenvolver ações eficientes e efetivas na prevenção e promoção de saúde dos usuários do SUS.

Em relação a novas propostas e ferramentas de atuação, como o protocolo colocado neste trabalho, ressalta-se a importância de vencer resistências por parte dos profissionais, o apoio da gestão para implementação das propostas e a educação continuada. Dessa forma, haverá capacidade de desenvolver uma prática profissional mais adequada aos modelos de atenção à saúde vigentes ao longo da evolução das políticas de saúde e perfil sociodemográfico da população.

\section{Referências}

1. Brasil ACO, Brandão JAM, Silva MON, Filho VCG. O papel do fisioterapeuta do programa saúde da família do município de Sobral-Ceará. RBPS. 2005; 18(1):3-6.

2. Ribeiro KSQ. A atuação da fisioterapia na atenção primária à saúde. Fisioterapia Brasil. 2002; 5(3):311318.

3. Resolução CNE/CES 4, de 19 de fevereiro de 2002. Institui Diretrizes Curriculares Nacionais do Curso de Graduação em Fisioterapia. Brasília, DF. Diário Oficial da União, 4 de março de 2002.

4. Portaria $\mathrm{GM} \mathrm{N}^{\circ}$ 154, 24 de janeiro de 2008. Criação dos Núcleos de Apoio à Saúde da Família. Brasília, DF. Diário Oficial da União, 04 mar 2008.

5. Organização Mundial da Saúde. Cuidados inovadores para condições crônicas: componentes estruturais de ação. Brasília; 2003.

6. Mendes EV. As redes de atenção à saúde. Belo Horizonte: Escola de Saúde Pública de Minas Gerais; 2009.

7. Ministério da Saúde. Endocrinologia e nefrologia. Protocolos de encaminhamento da atenção básica para a atenção especializada. Brasília: MS. [internet], 2016 [acesso em Jun 2016]; 25. Disponível em: http://www.saude.gov.br/bvs.

8. Farias N, Buchalla CM. A Classificação Internacional de Funcionalidade, Incapacidade e Saúde da Organização Mundial da Saúde: Conceitos, Usos e Perspectivas. Rev Bras Epidemiol. 2005; 8(2):187-93.

9. Bardin L. Análise de Conteúdo. 1a ed. São Paulo: Retórica; 2011.

10. Campos CJG. Método de análise de conteúdo: ferramenta para a análise de dados qualitativos no campo da saúde. Rev Bras Enferm. 2004; 57(5):611-614.

11. Camara AMCS. Oficina de Educação Interprofissional para a Residência Multiprofissional. Cad Edu Saude e Fis. 2014; (1):27-34.

12. Yonamine CY, Trelha CS. O modo de fazer saúde: a fisioterapia na residência multiprofissional em saúde da família em uma unidade básica. Rev. Espaço Saúde. 2009;11(1):17-27.

13. Silva DJ, Da Ros MA. Inserção de profissionais de fisioterapia na equipe de saúde da família e Sistema Único de Saúde: desafios na formação. Ciência \& Saúde Coletiva. 2007; 12(6):1673-1681.

14. Teixeira RC. Aderência dos cursos de Fisioterapia da região Norte às Diretrizes Curriculares Nacionais. Fisioter Mov. 2012; 25(1):47-54. 
15. Pain JS, Alves F, Naomar A. Saúde Coletiva: uma nova saúde pública ou campo aberto a novos paradigmas? Rev. Saúde Pública. 1998; 32(4):299-316.

16. Conselho Regional de Fisioterapia e Terapia Ocupacional da $5^{\mathrm{a}}$ região. Leis e atos normativos das profissões do fisioterapeuta e terapeuta ocupacional. Porto Alegre; 1997.

17. Augusto VG, Aquino CF, Machado NC, Cardoso VA, Ribeiro S. Promoção de saúde em unidades básicas: análise das representações sociais dos usuários sobre a atuação da fisioterapia. Cienc Saúde Coletiva 2011; 16(supl 1) 957:963.

18. Neves LMT, Acioli GG. Desafios da integralidade: revisitando as concepções sobre o papel do fisioterapeuta na equipe de Saúde da Família. Interface (Botucatu). 2011; 15(37):551-564.

19. Savassi, LCM. Qualidade em serviços públicos: os desafios da atenção primária. Rev bras med fam comunidade. 2012; 7(23):69-74.

20. Brasil. Lei $n^{\circ} 8080$, de 19 de setembro de 1990. Dispõe sobre as condições para a promoção, proteção e recuperação da saúde, a organização e o funcionamento dos serviços correspondentes e dá outras providências. Brasília, DF. Diário Oficial da União, 19 set. 1990.

21. Ministério da Saúde. Portaria $n^{\circ} 1.996$, de 20 de agosto de 2007. Dispõe sobre as diretrizes para a implementação da Política Nacional de Educação Permanente em Saúde. Brasília, DF: Ministério da Saúde; 2007.

22. Figueiredo EBL, Gouvêa MV, Cortez EA, Santos SCP, Alóchio KV, Silva, LM Alves. Dez anos da educação permanente como política de formação em saúde no brasil: um estudo das teses e dissertações. Trab Educ Saúde 2017; 15(1):147-162.

23. Silva LAA, Schmidt SMS, Noal HC, Signor E, Gomes IEM. Avaliação da educação permanente no processo de trabalho em saúde. Trab Educ Saúde 2016; 14(3):765-781.

24. Lima DLF. Movimento para a vida (mpav): proposta de um novo índice de Funcionalidade para o idoso. Rev. CIF Brasil. 2015; 3(3):28-39.

25. Belmonte LM, Chiaradia LCN, Belmonte LAO. CIF nos cursos de graduação de Fisioterapia da grande Florianópolis. Rev. CIF Brasil. 2015; 2(2):11-24.

26. Organização Mundial de Saúde /Organização Panamericana de Saúde. CIF classificação internacional de funcionalidade, incapacidade e saúde. São Paulo; 2003.

27. Sampaio RF, Mancini MC, Fonseca ST. Produção científica e atuação profissional: aspectos que limitam essa integração na fisioterapia e na terapia ocupacional. Rev Bras Fisioterapia. 2002; 6(3):113-118.

28. Brockow T, Cieza A, Kuhlow H, Sigl T, Franke T, Harder M. Identifying the concepts contained in outcome measures of clinical trials on musculoskeletal disorders and chronic widespread pain using the international classification of functioning, disability and health as a reference. J Rehabil Med. 2004; (44):30-36.

29. Steiner WA, Ryser L, Huber E, Uebelhart D, Aeschlimann A, Stucki G. Use of the ICF model as a clinical problem-solving tool in physical therapy and rehabilitation medicine. Phys Ther. 2002; 82(11):10981107.

30. Silva DJ, Ros MA. Inserção de profissionais de fisioterapia na equipe de saúde da família e Sistema Único de Saúde: desafios na formação. Ciência \& Saúde Coletiva. 2007; 12(6):1673-1681. 